A C G

publications organic communications

\title{
A simple, efficient synthesis and molecular docking studies
}

\section{of 2-styrylchromones}

\section{Priyadarsini Pullagura ${ }^{1}$, , Madhava Rao Vallabhaneni ${ }^{1}{ }^{*}$, \\ Hanumatha Rao Addanki ${ }^{-2}$, Subramanyam Chennamsetty ${ }^{1}$ and Ranganayakulu Yenisetty 3}

\author{
${ }^{I}$ Department Chemistry, Bapatla Engineering College,Bapatla-522102,A.P., India \\ ${ }^{2}$ Department Chemistry, KRK Govt Degree College, Addhanki-523201, A.P., India \\ ${ }^{3}$ Department Chemistry, Chalapati Institute of Engineering \& Technology, Guntur-522034, A.P., India
}

(Received February 08, 2021; Revised April 29, 2021; Accepted May 14, 2021)

\begin{abstract}
Styrylchromones have been synthesized successfully by using eco-friendly, recoverable and reusable PEG-400 as reaction medium in the presence of the catalytic amount of piperidine under warm conditions through 1,5-diphenylpenta-2,4-dien-1-ones as intermediates followed by oxidative cyclization with iodine in the same reaction vessel starting from 2-hydroxyacetophenones and cinnamaldehydes. The synthesized compounds were characterized by some physical methods and spectral data like IR, NMR, and LCMS etc. In silico molecular docking study was performed for all the synthesized compounds to know their ability in inhibiting pancreatic $\alpha$-amylase enzyme. In this study, the compounds $24,25,26 \& 28$ with binding energies, $-8.7,-8.8,-8.6$ and $-8.4 \mathrm{kcal} / \mathrm{mol}$ respectively were found to be more active amongst the synthesized when compared with standard drug, acarbose ($8.2 \mathrm{kcal} / \mathrm{mol})$.
\end{abstract}

Keywords: 2-Styrylchromones; eco-friendly; PEG-400; molecular docking; acarbose; $\alpha$-amylase. (C2021 ACG Publications. All right reserved.

\section{Introduction}

2-Styrylchromones are the new class of oxygen containing heterocyclic compounds, play an important role in nature due to their wide range of biological activities ${ }^{1-3}$ including pharmacological and biocidal activities ${ }^{4-7}$. These biological activities are due to the number and position of various substituents in the core structure which create great demand for their synthesis in past few years.

However, several attempts were made to synthesize ${ }^{8-15} 2$-styrylchromones, still there is a large scope for the efficient and eco-friendly routes to synthesize novel and potent bioactive analogues. This motivated many researchers as a result of which a large variety of 2-styrylchromones derivatives were reported $^{6,10,16-19}$ as potential therapeutic agents.

Chromone (benz- $\gamma$-pyrone or 4H-chromen-4-one) derivatives (Figure 1) with styryl group at $2^{\text {nd }}$ position have attracted a great deal of attention by virtue of their substantial anti-oxidant ${ }^{20}$, anti-cancer, ${ }^{21}$ anti-proliferal ${ }^{22}$, anti-tumor ${ }^{23}$ and anti-viral ${ }^{24}$ activities. Some research on chromone derivatives demonstrated their potentiality as anti-diabetic agents ${ }^{25,26}$.

\footnotetext{
*Corresponding author: E-Mail: vmrgpm@gmail.com, Phone: + 91-9440484238; 8374498399.
} 
All these widespread biological activities and scarcity of 2-styrylchromones in nature ${ }^{27,28}$ along with some others ${ }^{29-33}$ promoted us to synthesize a series of 2-styrylchromones from simple starting compounds with an efficient way and to screen them for their anti-diabetic activity using in silico molecular docking studies.<smiles>O=c1ccoc2ccccc12</smiles>

Chromone (Benz- $\gamma$-pyrone)

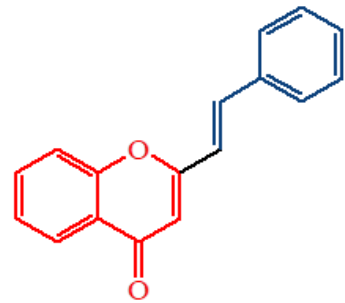

2-Styrylchromone

Figure 1. Structures of chromone and its derivative

\section{Experimental}

\subsection{Methods \& Materials:}

All chemicals needed for experimental part were purchased from National Scientific Products, Guntur. The melting point of all the synthesized compounds were determined in open capillary tubes using melting point apparatus. The synthesized compounds were characterized by various spectroscopic methods like FTIR, ${ }^{1} \mathrm{H} \&{ }^{13} \mathrm{C}$-NMR (Bruker, $400 \mathrm{MHz}$ ), LCMS and elemental analysis (Vario El-III). With the help of Marvin view software, the structures of all the compounds were drawn, optimized and converted them into required format. 1-Click docking software powered by Auto Dock Vina docking algorithm was used for in silico molecular docking study ${ }^{34,35}$.

\subsection{Synthetic Procedure for 2-Styrylchromones Preparation (20-31):}

The substituted acetophenone and cinnamaldehyde in 1:1 molar ratio were dissolved in $5 \mathrm{~mL}$ of PEG-400 and stirred at $40-50{ }^{\circ} \mathrm{C}$ with $0.4 \mathrm{~mL}$ of piperidine for about half an hour. The progress of the reaction was monitored by TLC using hexane:ethylacetate (9:1) as eluent. All the intermediate compounds were obtained in yellow colour.

The reaction intermediate (1,5-diphenylpenta-2,4-dien-1-one) thus obtained was dissolved in 5 $\mathrm{mL}$ of PEG in the same round bottom flask and a catalytic amount $(10 \mathrm{mg})$ of powdered iodine was added to it. The reaction mixture was refluxed in an oil bath with intermittent shaking for 3-4 hours at $\approx 140{ }^{\circ} \mathrm{C}$. Then the reaction mixture was cooled to room temperature, poured on to crushed ice with stirring and allowed the ice to melt. The chromone was filtered, washed with sodium thiosulfate solution until the washings were free from iodine, followed by with water. The product was dried and then purified using adsorption (column) chromatographic method using hexane:ethylacetate (9.5:0.5 and 9:1) as eluent and then recrystallized from ethanol.

The structures of all the synthesized compounds were characterized from their spectral data.

6-Hydroxy-2-(3, 4-dimethoxystyryl) chromone (20): Pale brown solid (285 mg, 82.3\%), m.p 208-210 ${ }^{\circ} \mathrm{C}$; IR (KBr) v $v_{\max }: 3435,2892,1621,1522,1227,1108,1032,829,775 \mathrm{~cm}^{-1} ;{ }^{1} \mathrm{H}-\mathrm{NMR}$ (DMSO-d 6 ): $\delta$ $9.87(1 \mathrm{H}, \mathrm{br}, \mathrm{s}, \mathrm{Ar}-\mathrm{OH}), 7.58(1 \mathrm{H}, \mathrm{d}, J=8.9 \mathrm{~Hz}, \mathrm{H}-8), 7.50\left(1 \mathrm{H}, \mathrm{d}, J=1.7 \mathrm{~Hz}, \mathrm{H}-2^{\prime}\right), 7.31(1 \mathrm{H}, \mathrm{d}, J=16.1$ $\mathrm{Hz}, \mathrm{H}-\beta), 7.19(1 \mathrm{H}, \mathrm{d}, J=2.8 \mathrm{~Hz}, \mathrm{H}-5), 7.06(1 \mathrm{H}, \mathrm{dd}, J=8.9,2.8 \mathrm{~Hz}, \mathrm{H}-7), 6.98(1 \mathrm{H}, \mathrm{dd}, J=8.1,1.7 \mathrm{~Hz}$, H-6'), $6.87(1 \mathrm{H}, \mathrm{d}, J=16.1 \mathrm{~Hz}, \mathrm{H}-\alpha), 6.73\left(1 \mathrm{H}, \mathrm{d}, J=8.1 \mathrm{~Hz}, \mathrm{H}-5^{\prime}\right), 6.30(1 \mathrm{H}, \mathrm{s}, \mathrm{H}-3), 3.83-3.92(6 \mathrm{H}, \mathrm{s}$, $\left.\mathrm{Ar}^{-} \mathrm{OCH}_{3}\right) ;{ }^{13} \mathrm{C}-\mathrm{NMR}\left(\left(\mathrm{DMSO}-\mathrm{d}_{6}\right): \delta 175.8(\mathrm{C}-4), 163.6(\mathrm{C}-2), 154.2(\mathrm{C}-6), 149.5(\mathrm{C}-3 \mathrm{\prime}), 149.3(\mathrm{C}-8 \mathrm{a})\right.$, 149.1 (C-4'), 136.5 (C- $\beta), 127.6\left(\mathrm{C}-1^{\prime}\right), 124.8$ (C-4a), 122.3(C-7), 121.8(C-6'), 119.1(C-8), 118.7 (C- $\left.\alpha\right)$, 115.4(C-5'), 114.6(C-5), 109.2(C-2'), 106.9(C-3) and 55.7(OMe-4'), 56.4(OMe-3'); LC-MS (ESI, negative ion mode), $m / z: 323[\mathrm{M}-\mathrm{H}]$; e elemental analysis for $\mathrm{C}_{19} \mathrm{H}_{16} \mathrm{O}_{5}$, found (calcd): $\mathrm{C} 70.35$ (70.37); H 5.01 (4.94).

6-Methoxy-2-(3, 4-dimethoxystyryl)chromone (21): Snuff color solid (287 mg, 84.9\%), m.p. 186-187 ${ }^{\circ} \mathrm{C}\left(\right.$ lit $\left.^{2}, 159-161^{\circ} \mathrm{C}\right)$; IR (KBr) v $v_{\max }: 2983,2835,1632,1527,1218,1110,830,781 \mathrm{~cm}^{-1}$; ${ }^{1} \mathrm{H}-\mathrm{NMR}$ 
(DMSO-d 6 ): $\delta 7.56(1 \mathrm{H}, \mathrm{d}, J=8.9 \mathrm{~Hz}, \mathrm{H}-8), 7.48\left(1 \mathrm{H}, \mathrm{d}, J=1.7 \mathrm{~Hz}, \mathrm{H}-2^{\prime}\right), 7.35(1 \mathrm{H}, \mathrm{d}, J=16.2 \mathrm{~Hz}, \mathrm{H}-\beta)$, $7.32(1 \mathrm{H}, \mathrm{d}, J=2.8 \mathrm{~Hz}, \mathrm{H}-5), 7.16(1 \mathrm{H}, \mathrm{dd}, J=8.9,2.8 \mathrm{~Hz}, \mathrm{H}-7), 6.98\left(1 \mathrm{H}, \mathrm{dd}, J=8.1,1.7 \mathrm{~Hz}, \mathrm{H}-6{ }^{\prime}\right), 6.85$ $(1 \mathrm{H}, \mathrm{d}, J=16.2 \mathrm{~Hz}, \mathrm{H}-\alpha), 6.71\left(1 \mathrm{H}, \mathrm{d}, J=8.1 \mathrm{~Hz}, \mathrm{H}-5^{\prime}\right), 6.31(1 \mathrm{H}, \mathrm{s}, \mathrm{H}-3), 3.83\left(6 \mathrm{H}, \mathrm{s}, \mathrm{Ar}-\mathrm{OCH}_{3}\right), 3.67(3 \mathrm{H}$, s, $\left.\mathrm{Ar}-\mathrm{OCH}_{3}\right) ;{ }^{13} \mathrm{C}-\mathrm{NMR}\left(\left(\mathrm{DMSO}-\mathrm{d}_{6}\right): \delta 175.6(\mathrm{C}-4), 163.6(\mathrm{C}-2), 156.3(\mathrm{C}-6), 149.8(\mathrm{C}-3\right.$ '), $149.4(\mathrm{C}-8 \mathrm{a})$, 149.2(C-4'), 135.8(C- $\beta), 128.3\left(\mathrm{C}^{\prime} 1^{\prime}\right), 125.2(\mathrm{C}-4 \mathrm{a}), 121.7(\mathrm{C}-7), 119.8\left(\mathrm{C}-6{ }^{\prime}\right), 119.3(\mathrm{C}-8), 118.9(\mathrm{C}-\alpha)$, 115.6 (C-5'), 115.2(C-5), 111.3(C-2'), 106.7(C-3) and 55.8 (OMe-4',6), 56.2(OMe-3'); LC-MS (ESI, negative ion mode): $\mathrm{m} / \mathrm{z}: 337$ [M-H] ; elemental analysis: for $\mathrm{C}_{20} \mathrm{H}_{18} \mathrm{O}_{5}$, found (calcd): $\mathrm{C}$ 70.97(71.00); H 5.35(5.32).

7-Hydroxy-2-(3, 4-dimethoxystyryl)chromone (22): Brick red solid (265 mg, 81.7\%), m.p. 223-225 ${ }^{\circ} \mathrm{C}$; $\operatorname{IR}(\mathrm{KBr}) v_{\max }: 3429,2924,1629,1608,1458,1225,1131,865 \mathrm{~cm}^{-1} ;{ }^{1} \mathrm{H}-\mathrm{NMR}$ (DMSO-d 6 ): $\delta 9.67(1 \mathrm{H}$, br, s, Ar-OH), $7.78(1 \mathrm{H}, \mathrm{d}, J=8.6 \mathrm{~Hz}, \mathrm{H}-5), 7.36(1 \mathrm{H}, \mathrm{d}, J=16.1 \mathrm{~Hz}, \mathrm{H}-\beta), 7.13\left(1 \mathrm{H}, \mathrm{d}, J=2.0 \mathrm{~Hz}, \mathrm{H}-2^{\prime}\right)$, $6.98\left(1 \mathrm{H}, \mathrm{dd}, J=8.2,2.0 \mathrm{~Hz}, \mathrm{H}-6^{\prime}\right), 6.88(1 \mathrm{H}, \mathrm{d}, J=16.1 \mathrm{~Hz}, \mathrm{H}-\alpha), 6.65(1 \mathrm{H}, \mathrm{d}, J=2.2 \mathrm{~Hz}, \mathrm{H}-8), 6.52(1 \mathrm{H}$, $\mathrm{dd}, J=8.6,2.2 \mathrm{~Hz}, \mathrm{H}-6), 6.78\left(1 \mathrm{H}, \mathrm{d}, J=8.2 \mathrm{~Hz}, \mathrm{H}-5^{\prime}\right), 6.29(1 \mathrm{H}, \mathrm{s}, \mathrm{H}-3), 3.88\left(3 \mathrm{H}, \mathrm{s}, \mathrm{Ar}-\mathrm{OCH}_{3}\right), 3.67(3 \mathrm{H}$, s, $\left.\mathrm{Ar}-\mathrm{OCH}_{3}\right) ;{ }^{13} \mathrm{C}-\mathrm{NMR}\left(\left(\mathrm{DMSO}-\mathrm{d}_{6}\right): \delta 176.8(\mathrm{C}-4), 164.3(\mathrm{C}-7), 162.4(\mathrm{C}-2), 156.2(\mathrm{C}-8 \mathrm{a}), 149.3(\mathrm{C}-3\right.$ '), 148.8(C-4'), 136.5(C- $\beta), 134.7(\mathrm{C}-5), 127.3\left(\mathrm{C}-1^{\prime}\right), 120.7\left(\mathrm{C}-6^{\prime}\right), 116.8(\mathrm{C}-\alpha), 116.3\left(\mathrm{C}-5{ }^{\prime}\right), 115.8(\mathrm{C}-4 \mathrm{a})$, 113.7(C-2'), 112.5(C-6), 107.6(C-3), 103.8(C-8), and 56.3(OMe-4'), 55.8(OMe-3'); LC-MS (ESI negative ion mode): $\mathrm{m} / \mathrm{z}: 323[\mathrm{M}-\mathrm{H}]$; elemental analysis for $\mathrm{C}_{19} \mathrm{H}_{16} \mathrm{O}_{5}$, found (calcd): $\mathrm{C} 70.98$; (70.37); H 5.40 (4.94).

7-Methoxy-2-(3, 4-dimethoxystyryl)chromone (23): Yellowish orange solid (285 mg, 84.3\%), m.p. 179$181{ }^{\circ} \mathrm{C}\left(\right.$ lit $\left.^{2}, 198-199{ }^{\circ} \mathrm{C}\right)$; IR(KBr) $v_{\max }: 2933,2835,1634,1605,1468,1242,1162,847 \mathrm{~cm}^{-1} ;{ }^{1} \mathrm{H}-\mathrm{NMR}$ (DMSO-d $\left.)_{6}\right): \delta 7.86(1 \mathrm{H}, \mathrm{d}, J=8.6 \mathrm{~Hz}, \mathrm{H}-5), 7.38(1 \mathrm{H}, \mathrm{d}, J=16.3 \mathrm{~Hz}, \mathrm{H}-\beta), 7.15\left(1 \mathrm{H}, \mathrm{d}, J=2.0 \mathrm{~Hz}, \mathrm{H}-2^{\prime}\right)$, $7.02\left(1 \mathrm{H}, \mathrm{dd}, J=8.2,2.0 \mathrm{~Hz}, \mathrm{H}-6^{\prime}\right), 6.89(1 \mathrm{H}, \mathrm{d}, J=16.3 \mathrm{~Hz}, \mathrm{H}-\alpha), 6.77\left(1 \mathrm{H}, \mathrm{d}, J=8.2 \mathrm{~Hz}, \mathrm{H}-5^{\prime}\right), 6.69$ $(1 \mathrm{H}, \mathrm{d}, J=2.2 \mathrm{~Hz}, \mathrm{H}-8), 6.55(1 \mathrm{H}, \mathrm{dd}, J=8.6,2.2 \mathrm{~Hz}, \mathrm{H}-6), 6.31(1 \mathrm{H}, \mathrm{s}, \mathrm{H}-3), 3.90-3.86(6 \mathrm{H}, \mathrm{s}, \mathrm{Ar}-$ $\left.\mathrm{OCH}_{3}\right), 3.65\left(3 \mathrm{H}, \mathrm{s}, \mathrm{Ar}-\mathrm{OCH}_{3}\right) .{ }^{13} \mathrm{C}-\mathrm{NMR}\left(\left(\mathrm{DMSO}-\mathrm{d}_{6}\right): \delta 176.5(\mathrm{C}-4), 165.8(\mathrm{C}-7), 162.6(\mathrm{C}-2), 156.7(\mathrm{C}-\right.$ 8a), 149.4 (C-3'), 149.1(C-4'), 136.7(C- $\beta), 134.9(\mathrm{C}-5), 127.6\left(\mathrm{C}-1^{\prime}\right), 121.8\left(\mathrm{C}-66^{\prime}\right), 117.2(\mathrm{C}-\alpha), 116.6(\mathrm{C}-$ 5'), 115.4(C-4a), 113.4 (C-2'), 112.2(C-6), 107.3(C-3), 103.2(-C8), and 56.8 (OMe-4'7), 55.6(OMe-3'); LC-MS (ESI negattive ion mode): $\mathrm{m} / z: 337$ [M-H]; elemental analysis for $\mathrm{C}_{20} \mathrm{H}_{18} \mathrm{O}_{5}$, found (calcd): $\mathrm{C}$ 70.28(71.00); H 5.37(5.32).

7-Hydroxy-2-(4-hydroxy-3-methoxystyryl)chromone (24): Brick red solid (252 mg, 80.7\%), m.p. 258$260{ }^{\circ} \mathrm{C}\left(\mathrm{lit}{ }^{44}, 260-262{ }^{\circ} \mathrm{C}\right)$; IR(KBr) v $v_{\max }: 3365,2918,1636,1618,1564,1468,1328,1272,1169,836$ $\mathrm{cm}^{-1} ;{ }^{1} \mathrm{H}-\mathrm{NMR}$ (DMSO-d $\left.\mathrm{d}_{6}\right): \delta 9.67(1 \mathrm{H}, \mathrm{br}, \mathrm{s}, \mathrm{Ar}-\mathrm{OH}), 8.85(1 \mathrm{H}, \mathrm{br}, \mathrm{s}, \mathrm{Ar}-\mathrm{OH}), 7.83(1 \mathrm{H}, \mathrm{d}, J=8.6 \mathrm{~Hz}$, H-5), $7.55(1 \mathrm{H}, \mathrm{d}, J=16.0 \mathrm{~Hz}, \mathrm{H}-\beta), 7.12\left(1 \mathrm{H}, \mathrm{d}, J=2.0 \mathrm{~Hz}, \quad \mathrm{H}-2^{\prime}\right), 7.02\left(1 \mathrm{H}, \mathrm{dd}, J=8.2,2.0 \mathrm{~Hz}, \mathrm{H}-6^{\prime}\right)$, $6.94(1 \mathrm{H}, \mathrm{d}, J=16.0 \mathrm{~Hz}, \mathrm{H}-\alpha), 6.87(1 \mathrm{H}, \mathrm{d}, J=2.2 \mathrm{~Hz}, \mathrm{H}-8), 6.85$ (1H, dd, $J=8.6,2.2 \mathrm{~Hz}, \mathrm{H}-6), 6.82(1 \mathrm{H}$, $\left.\mathrm{d}, J=8.2 \mathrm{~Hz}, \mathrm{H}-5^{\prime}\right), 6.23(1 \mathrm{H}, \mathrm{s}, \mathrm{H}-3), 3.85\left(3 \mathrm{H}, \mathrm{s}, \mathrm{Ar}-\mathrm{OCH}_{3}\right) ;{ }^{13} \mathrm{C}-\mathrm{NMR}\left(\left(\mathrm{DMSO}-\mathrm{d}_{6}\right): \delta 176.2(\mathrm{C}-4)\right.$, 163.1 (C-7), 162.2(C-2), 157.1(C-8a), 148.5(C-3'), 148.2 (C-4'), 136.4(C- $\beta), 126.9(\mathrm{C}-5), 126.3\left(\mathrm{C}-1^{\prime}\right)$, 122.4(C-6'), 117.6 (C- $\alpha), 116.3(\mathrm{C}-5$ '), 115.5(C-4a), 114.6(C-2'), 113.4 (C-6), 108.7(C-3), 102.3(C-8), and 55.8(OMe-3'); LC-MS (ESI, negative ion mode): $m / z: 309\left([\mathrm{M}-\mathrm{H}]^{-}\right.$; elemental analysis for $\mathrm{C}_{18} \mathrm{H}_{14} \mathrm{O}_{5}$ : found (calcd): C 69.65 (69.68); H, 4.6 (4.5).

7-Methoxy-2-(4-hydroxy-3-methoxystyryl)chromone (25): Brown solid (270 mg, 83.3\%), m.p. 227-229 ${ }^{\circ} \mathrm{C}$; IR(KBr) $v_{\max }: 3303,2919,1628,1609,1535,1448,1219,1017,771 \mathrm{~cm}^{-1} ;{ }^{1} \mathrm{H}-\mathrm{NMR}\left(\mathrm{DMSO}-\mathrm{d}_{6}\right): \delta$ $9.07(1 \mathrm{H}, \mathrm{br}, \mathrm{s}, \mathrm{Ar}-\mathrm{OH}), 7.72(1 \mathrm{H}, \mathrm{d}, J=8.6 \mathrm{~Hz}, \mathrm{H}-5), 7.57(1 \mathrm{H}, \mathrm{d}, J=16.1 \mathrm{~Hz}, \mathrm{H}-\beta), 7.15(1 \mathrm{H}, \mathrm{d}, J=2.0$ $\left.\mathrm{Hz}, \quad \mathrm{H}-2^{\prime}\right), 7.03\left(1 \mathrm{H}, \mathrm{dd}, J=8.2,2.0 \mathrm{~Hz}, \mathrm{H}-6^{\prime}\right), 6.93(1 \mathrm{H}, \mathrm{d}, J=16.1 \mathrm{~Hz}, \mathrm{H}-\alpha), 6.86(1 \mathrm{H}, \mathrm{d}, J=2.2 \mathrm{~Hz}$, $\mathrm{H}-8), 6.84(1 \mathrm{H}, \mathrm{dd}, J=8.6,2.2 \mathrm{~Hz}, \mathrm{H}-6), 6.80\left(1 \mathrm{H}, \mathrm{d}, J=8.2 \mathrm{~Hz}, \mathrm{H}-5^{\prime}\right), 6.27(1 \mathrm{H}, \mathrm{s}, \mathrm{H}-3), 3.83-3.85(6 \mathrm{H}$, s, $\left.\mathrm{Ar}-\mathrm{OCH}_{3}\right) ;{ }^{13} \mathrm{C}-\mathrm{NMR}\left(\left(\mathrm{DMSO}_{6} \mathrm{~d}_{6}\right): \delta 175.9(\mathrm{C}-4), 163.7(\mathrm{C}-7), 161.9(\mathrm{C}-2), 157.3(\mathrm{C}-8 \mathrm{a}), 149.5\left(\mathrm{C}-3^{\prime}\right)\right.$, 148.0(C-4'), 136.4(C- $\beta), 126.8(\mathrm{C}-5), 126.4$ (C-1'), 122.5(C-6'), 118.1(C- $\alpha), 116.3\left(\mathrm{C}-5^{\prime}\right), 115.2$ (C-4a), 114.9(C-2'), 114.0(C-6), 107.9(C-3), 101.9(C-8), and 55.7-55.9(OMe-3',7); LC-MS (ESI, negative ion mode): $m / z: 323$ [M-H]; ; elemental analysis for $\mathrm{C}_{19} \mathrm{H}_{16} \mathrm{O}_{5}$ : found (calcd): $\mathrm{C}, 70.36(70.37) ; \mathrm{H}, 4.97$ (4.93).

6-Hydroxy-2-(4-hydroxy-3-methoxystyryl)chromone (26): Light yellow solid (255 mg, 82.2\%), m.p. 265-267 ${ }^{\circ} \mathrm{C}\left(\mathrm{lit}^{44}, 268-270{ }^{\circ} \mathrm{C}\right)$. IR (KBr) v max $: 3454,2986,1636,1591,1535,1463,1269,1204,1023$, $835 \mathrm{~cm}^{-1}$; ${ }^{1} \mathrm{H}-\mathrm{NMR}$ (DMSO-d 6 ): $\delta 9.89(1 \mathrm{H}, \mathrm{br}, \mathrm{s}, \mathrm{Ar}-\mathrm{OH}), \delta 9.55(1 \mathrm{H}, \mathrm{br}, \mathrm{s}, \mathrm{Ar}-\mathrm{OH}), 7.54(1 \mathrm{H}, \mathrm{d}, J=8.9$ $\mathrm{Hz}, \mathrm{H}-8), 7.53\left(1 \mathrm{H}, \mathrm{d}, J=1.7 \mathrm{~Hz}, \mathrm{H}-2^{\prime}\right), 7.27(1 \mathrm{H}, \mathrm{d}, J=16.0 \mathrm{~Hz}, \mathrm{H}-\beta), 7.26(1 \mathrm{H}, \mathrm{d}, J=2.8 \mathrm{~Hz}, \mathrm{H}-5), 7.23$ $(1 \mathrm{H}, \mathrm{dd}, J=8.9,2.8 \mathrm{~Hz}, \mathrm{H}-7), 7.10\left(1 \mathrm{H}, \mathrm{dd}, J=8.1,1.7 \mathrm{~Hz}, \mathrm{H}-6^{\prime}\right), 7.02(1 \mathrm{H}, \mathrm{d}, J=16.0 \mathrm{~Hz}, \mathrm{H}-\alpha), 6.80$ $\left(1 \mathrm{H}, \mathrm{d}, J=8.1 \mathrm{~Hz}, \mathrm{H}-5^{\prime}\right), 6.29(1 \mathrm{H}, \mathrm{s}, \mathrm{H}-3), 3.85\left(3 \mathrm{H}, \mathrm{s}, \mathrm{Ar}-\mathrm{OCH}_{3}\right) ;{ }^{13} \mathrm{C}-\mathrm{NMR}\left(\left(\mathrm{DMSO}-\mathrm{d}_{6}\right): \delta 176.5(\mathrm{C}-\right.$ 
4), 162.2 (C-2), 154.5(C-6), 150.2(C-8a), 149.2(C-3'), 148.3(C-4'), 136.5(C- $\beta), 126.3\left(\mathrm{C}-1^{\prime}\right), 123.9(\mathrm{C}-$ 4a), 123.1(C-7), 122.1 (C-6'), 118.8(C-8), 117.5(C- $\alpha), 115.6\left(\mathrm{C}-5^{\prime}\right), 111.2(\mathrm{C}-5), 108.5\left(\mathrm{C}-2^{\prime}\right), 107.9(\mathrm{C}-3)$ and 55.8(OMe-3'); LC-MS (ESI, negative ion mode): $\mathrm{m} / \mathrm{z}: 309$ [M-H]'; elemental analysis for $\mathrm{C}_{18} \mathrm{H}_{14} \mathrm{O}_{5}$ : found (calcd): C 69.62 (69.68); H 4.3 (4.5).

6-Methoxy-2-(4-hydroxy-3-methoxystyryl)chromone (27): Greenish yellow color solid (272 mg, 83.9\%), m.p. $231-233{ }^{\circ} \mathrm{C}\left(\mathrm{lit}^{44}, 230-232{ }^{\circ} \mathrm{C}\right)$; IR (KBr) v $v_{\max }: 3238,1641,1608,1530,1380,1273,1132,827 \mathrm{~cm}^{-}$ '; ${ }^{1} \mathrm{H}-\mathrm{NMR}$ (DMSO-d 6 ) : $\delta 9.54(1 \mathrm{H}, \mathrm{br}, \mathrm{s}, \mathrm{Ar}-\mathrm{OH}), 7.65(1 \mathrm{H}, \mathrm{d}, J=9.0 \mathrm{~Hz}, \mathrm{H}-8), 7.59(1 \mathrm{H}, \mathrm{d}, J=16.1$ $\mathrm{Hz}, \mathrm{H}-\beta), 7.55\left(1 \mathrm{H}, \mathrm{d}, J=2.2 \mathrm{~Hz}, \mathrm{H}-2^{\prime}\right), 7.38-7.31(2 \mathrm{H}, \mathrm{m}, \mathrm{H}-5$ \& 7$), 7.12(1 \mathrm{H}, \mathrm{dd}, J=8.1,2.2 \mathrm{~Hz}, \mathrm{H}-$ 6') $7.03(1 \mathrm{H}, \mathrm{d}, J=16.1 \mathrm{~Hz}, \mathrm{H}-\alpha), 6.80\left(1 \mathrm{H}, \mathrm{d}, J=8.1 \mathrm{~Hz}, \mathrm{H}-5^{\prime}\right), 6.35(1 \mathrm{H}, \mathrm{s}, \mathrm{H}-3), 3.86(6 \mathrm{H}, \mathrm{s}, 2 \mathrm{Ar}-$ $\left.\mathrm{OCH}_{3}\right) ;{ }^{13} \mathrm{C}-\mathrm{NMR}\left(\left(\mathrm{DMSO}-\mathrm{d}_{6}\right): \delta\right.$ 176.7(C-4), 163.8(C-2), 156.3(C-6), 150.4(C-3'), 149.2(C-8a), 148.1(C-4'), 137.2(C- $\beta), 126.8\left(\mathrm{C}-1^{\prime}\right), 124.5(\mathrm{C}-4 \mathrm{a}), 123.1(\mathrm{C}-7), 122.6$ (C-6'), 120.2(C- $\left.\alpha\right), 117.3(\mathrm{C}-8)$, 115.9 (C-5'), 111.3(C-5), 108.7(C-2'), 105.2(C-3) and 55.9 (OMe-3',6); LC-MS (ESI, negative ion mode): $m / z: 323[\mathrm{M}-\mathrm{H}]$; ; elemental analysis for $\mathrm{C}_{19} \mathrm{H}_{16} \mathrm{O}_{5}$ : found (calcd): $\mathrm{C}, 70.33$ (70.37); $\mathrm{H}, 5.00$ (4.94).

7-Hydroxy -2-(4-methoxystyryl)chromone (28): Orange color solid (240 mg, 81.6\%), m.p. 250-253 ${ }^{\circ} \mathrm{C}$ (lit $\left.{ }^{11}, 248-251^{\circ} \mathrm{C}\right)$. IR (KBr) $v_{\max }: 3431,2956,2833,1635,1609,1513,1465,1234,1137,843,813,765$ $\mathrm{cm}^{-1} ;{ }^{1} \mathrm{H}-\mathrm{NMR}\left(\mathrm{DMSO}-\mathrm{d}_{6}\right): \delta 9.76(1 \mathrm{H}, \mathrm{br}, \mathrm{s}, \mathrm{Ar}-\mathrm{OH}) ; 7.84(1 \mathrm{H}, \mathrm{d}, J=8.7 \mathrm{~Hz}, \mathrm{H}-5) ; 7.54(1 \mathrm{H}, \mathrm{d}, J=16.0$ $\mathrm{Hz} ; \mathrm{H}-\beta) ; 7.51\left(2 \mathrm{H}, \mathrm{d}, J=8.6 \mathrm{~Hz}, \mathrm{H}-2^{\prime}, 6^{\prime}\right) ; 6.97(1 \mathrm{H}, \mathrm{d}, J=16.0 \mathrm{~Hz}, \mathrm{H}-\alpha) ; 6.88(1 \mathrm{H}, \mathrm{d}, J=2.2 \mathrm{~Hz}, \mathrm{H}-8)$; $6.85(1 \mathrm{H}, \mathrm{dd}, J=8.6,2.2 \mathrm{~Hz}-\mathrm{H}-6) ; 6.82\left(2 \mathrm{H}, \mathrm{d}, J=8.6 \mathrm{~Hz}, \mathrm{H}-3^{\prime}, 5^{\prime}\right) ; 6.25(1 \mathrm{H}, \mathrm{s}, \mathrm{H}-3) ; 3.85\left(3 \mathrm{H}, \mathrm{s} . \mathrm{Ar}^{-\mathrm{OCH}_{3}}\right)$; ${ }^{13} \mathrm{C}-\mathrm{NMR}\left(\left(\mathrm{DMSO}-\mathrm{d}_{6}\right): \delta\right.$ 176.3(C-4), 164.8(C-7), 161.8(C-2), $158.0(\mathrm{C}-8 \mathrm{a}), 157.5(\mathrm{C}-4$ '), $136.5(\mathrm{C}-\beta)$, 130.2(C-5), 123.3(C-1'), 125.5(C-2',6'), 117.6(C $\left.\mathrm{C}_{\alpha}\right), 115.3\left(\mathrm{C}-3^{\prime}, 5^{\prime}\right), 114.6(\mathrm{C}-4 \mathrm{a}), 108.7$ (C-6), 106.3(C3), 103.3(C-8), and 55.8 (OMe-4'); LCMS (ESI, negative ion mode): $\mathrm{m} / \mathrm{z}: 293$ [M-H]; elemental analysis: for $\mathrm{C}_{18} \mathrm{H}_{14} \mathrm{O}_{4}$ : found (calcd): $\mathrm{C} 73.23$ (73.46); $\mathrm{H} 4.75(4.76)$.

7-Methoxy-2-(4-methoxystyryl)chromone (29): Yellowish orange solid (255 mg, 82.8\%), m.p. 172-175 ${ }^{\circ} \mathrm{C}\left(\mathrm{lit}{ }^{42,}, 140-141{ }^{\circ} \mathrm{C}\right.$ ); IR (KBr) v $v_{\max }: 2935,2819,1623,1605,1511,1436,1233,1125,812,763 \mathrm{~cm}^{-1}$; ${ }^{1} \mathrm{H}-\mathrm{NMR}\left(\right.$ DMSO-d $\left._{6}\right): \delta 7.74(1 \mathrm{H}, \mathrm{d}, J=8.8 \mathrm{~Hz}, \mathrm{H}-5) ; 7.57(1 \mathrm{H}, \mathrm{d}, J=16.1 \mathrm{~Hz} ; \mathrm{H}-\beta) ; 7.55(2 \mathrm{H}, \mathrm{d}, J=8.6$ $\left.\mathrm{Hz}, \mathrm{H}-2^{\prime}, 6^{\prime}\right) ; 6.88(1 \mathrm{H}, \mathrm{d}, J=16.1 \mathrm{~Hz}, \mathrm{H}-\alpha) ; 6.91(1 \mathrm{H}, \mathrm{d}, J=2.2 \mathrm{~Hz}, \mathrm{H}-8) ; 6.89(1 \mathrm{H}$, dd, $J=8.6,2.2 \mathrm{~Hz}-\mathrm{H}-$ 6); $6.85\left(2 \mathrm{H}, \mathrm{d}, J=8.6 \mathrm{~Hz}, \mathrm{H}-3^{\prime}, 5^{\prime}\right) ; 6.29(1 \mathrm{H}, \mathrm{s}, \mathrm{H}-3) ; 3.85\left(6 \mathrm{H}, \mathrm{s}, \mathrm{Ar}_{-} \mathrm{OCH}_{3}\right) ;{ }^{13} \mathrm{C}-\mathrm{NMR}\left(\left(\mathrm{DMSO}-\mathrm{d}_{6}\right): \delta\right.$ 176.2 (C-4), 165.6(C-7), 162.1(C-2), 157.9(C-8a), 157.6 (C-4'), 136.7 (C- $\beta), 129.8(\mathrm{C}-5), 123.4\left(\mathrm{C}-1^{\prime}\right)$, 125.7(C-2',6'), $118.2(\mathrm{C}-\alpha), 116.6\left(\mathrm{C}-3^{\prime}, 5^{\prime}\right), 114.8(\mathrm{C}-4 \mathrm{a}), 110.8(\mathrm{C}-6), 106.6(\mathrm{C}-3), 102.7(\mathrm{C}-8)$, and 56.2 (OMe-4',7); LCMS (ESI, negative ion mode): $m / z: 307$ [M-H]-; elemental analysis for $\mathrm{C}_{19} \mathrm{H}_{16} \mathrm{O}_{4}$ : found (calcd): C-74.01(74.02); H-5.23, (5.19).

6-hydroxy-2-(4-methoxystyryl)chromone (30): Brown color solid (240 mg, 81.6\%). m.p. 256-257 ${ }^{\circ} \mathrm{C}$ (lit ${ }^{41}, 257-259^{\circ} \mathrm{C}$ ); IR (KBr) v $v_{\max }: 3345,2936,1631,1617,1465,1247,1167 \mathrm{~cm}^{-1}$; ${ }^{1} \mathrm{H}-\mathrm{NMR}$ (DMSO$\left.\mathrm{d}_{6}\right): \delta 9.82(1 \mathrm{H}, \mathrm{br}, \mathrm{s}, \mathrm{Ar}-\mathrm{OH}), 7.67(1 \mathrm{H}, \mathrm{d}, J=8.5 \mathrm{~Hz}, \mathrm{H}-8), 7.55(1 \mathrm{H}, \mathrm{d}, J=16.2 \mathrm{~Hz}, \mathrm{H}-\beta), 7.42(2 \mathrm{H}, \mathrm{d}$, $\left.J=8.4 \mathrm{~Hz}, \mathrm{H}-2^{\prime}, 6^{\prime}\right), 7.30(2 \mathrm{H}, \mathrm{m}, \mathrm{H}-5,7), 6.91(1 \mathrm{H}, \mathrm{d}, J=16.2 \mathrm{~Hz}, \mathrm{H}-\alpha), 6.82\left(2 \mathrm{H}, \mathrm{d}, J=8.4 \mathrm{~Hz}, \mathrm{H}-3^{\prime}, 5^{\prime}\right)$, $6.38(1 \mathrm{H}, \mathrm{s}, \mathrm{H}-3), 3.89\left(3 \mathrm{H}, \mathrm{s}, \mathrm{OCH}_{3}\right) ;{ }^{13} \mathrm{C}-\mathrm{NMR}\left(\left(\mathrm{DMSO}_{-} \mathrm{d}_{6}\right): \delta 176.6(\mathrm{C}-4), 162.8(\mathrm{C}-2), 158.3\left(\mathrm{C}-4^{\prime}\right)\right.$, 149.7(C-6), 148.6(C-8a), 136.8(C- $\beta), 126.8\left(\mathrm{C}^{2} 2^{\prime}, 6^{\prime}\right), 125.3$ (C-1'), 123.8(C-4a), 123.7(C-7), 118.8(C-8), 117.3(C- $\alpha), 115.1\left(\mathrm{C}-3^{\prime}, 5^{\prime}\right), 113.2(\mathrm{C}-5), 107.3(\mathrm{C}-3)$, and 55.7 (OMe-4'); LCMS (ESI, negative ion mode): $\mathrm{m} / z: 293$ [M-H] ; elemental analysis for $\mathrm{C}_{18} \mathrm{H}_{14} \mathrm{O}_{4}$ : found (calcd): $\mathrm{C}, 73.44$ (73.46); H, 4.73 (4.76).

6-methoxy-2-(4-methoxystyryl)chromone (31): Pale yellow color solid (258 mg, 83.7\%); m.p. 167-169 ${ }^{\circ} \mathrm{C}\left(\mathrm{lit}^{41}, 167-169^{\circ} \mathrm{C}\right)$; IR (KBr) v $\mathrm{vax}_{\max }: 2985,2838,1632,1607,1565,1436,1243,1168 \mathrm{~cm}^{-1}$; ${ }^{1} \mathrm{H}-\mathrm{NMR}$ (DMSO-d $)_{6}$ : $\delta 7.73(1 \mathrm{H}, \mathrm{d}, J=8.7 \mathrm{~Hz}, \mathrm{H}-8), 7.58(1 \mathrm{H}, \mathrm{d}, J=16.3 \mathrm{~Hz}, \mathrm{H}-\beta), 7.51\left(2 \mathrm{H}, \mathrm{d}, J=8.3 \mathrm{~Hz}, \mathrm{H}-2^{\prime}, 6^{\prime}\right)$, 7.42-7.33(2H, m, H-5,7), 7.02(1H, d, $J=16.3 \mathrm{~Hz}, \mathrm{H}-\alpha), 6.80\left(2 \mathrm{H}, \mathrm{d}, J=8.3 \mathrm{~Hz}, \mathrm{H}-3^{\prime}, 5^{\prime}\right), 6.34(1 \mathrm{H}, \mathrm{s}, \mathrm{H}-$ 3), 3.93(3H, s, $\left.\mathrm{OCH}_{3}\right), 3.85\left(3 \mathrm{H}, \mathrm{s}, \mathrm{OCH}_{3}\right) ;{ }^{13} \mathrm{C}-\mathrm{NMR}\left(\left(\mathrm{DMSO}_{6} \mathrm{~d}_{6}\right): \delta 175.9(\mathrm{C}-4), 163.5(\mathrm{C}-2), 159.1(\mathrm{C}-\right.$ 4'), 151.9(C-6), 149.2(C-8a), 136.5(C- $\beta), 126.9\left(\mathrm{C}-2^{\prime}, 6^{\prime}\right), 125.6\left(\mathrm{C}-1^{\prime}\right), 123.5(\mathrm{C}-4 \mathrm{a}), 122.6(\mathrm{C}-7), 118.4$ (C-8), 117.8(C- $\alpha$ ), 114.9(C-3',5'), 113.6(C-5), 106.8(C-3), and 55.8 (OMe-4',6); LCMS (ESI, negative ion mode): $m / z: 307$ ([M-H]; elemental analysis for $\mathrm{C}_{19} \mathrm{H}_{16} \mathrm{O}_{4}$ : found (calcd): $\mathrm{C} 73.98$ (74.01); $\mathrm{H} 5.21$ (5.23). 


\subsection{In Silico Molecular Docking Studies with $\alpha$-Amylase}

Molecular docking studies of the prepared compounds were performed against $\alpha$-amylase enzyme, in order to understand the possible binding mechanism prior to their synthesis. Marvin view software was utilized to optimize the structures of the title compounds and the standard drug acarbose. In order to reveal the binding modes of the title compounds, docking simulation was performed targeting the crystal structure of pancreatic alpha amylase which was retrieved from RCSB, Protein Data Bank (PDB ID: 3IJ8) and the structure was optimized by removing the water molecules, hetero atoms, and co-factors. Hydrogen bonds, missing atoms, and charges were computed. The PDB structures of target protein (A) and standard drug, acarbose (B) were shown in Figure 1. The molecular docking method was performed using 1-click docking online server tool, which uses a Vina filter and dock multiple ligands into a single target with default binding site X: 7.2178, Y: 16.2957, Z: 42.1167. The docked process and interactions of compounds and protein were analyzed by using Discovery studio Visualizer ${ }^{36}$ V16.1.0.15350.
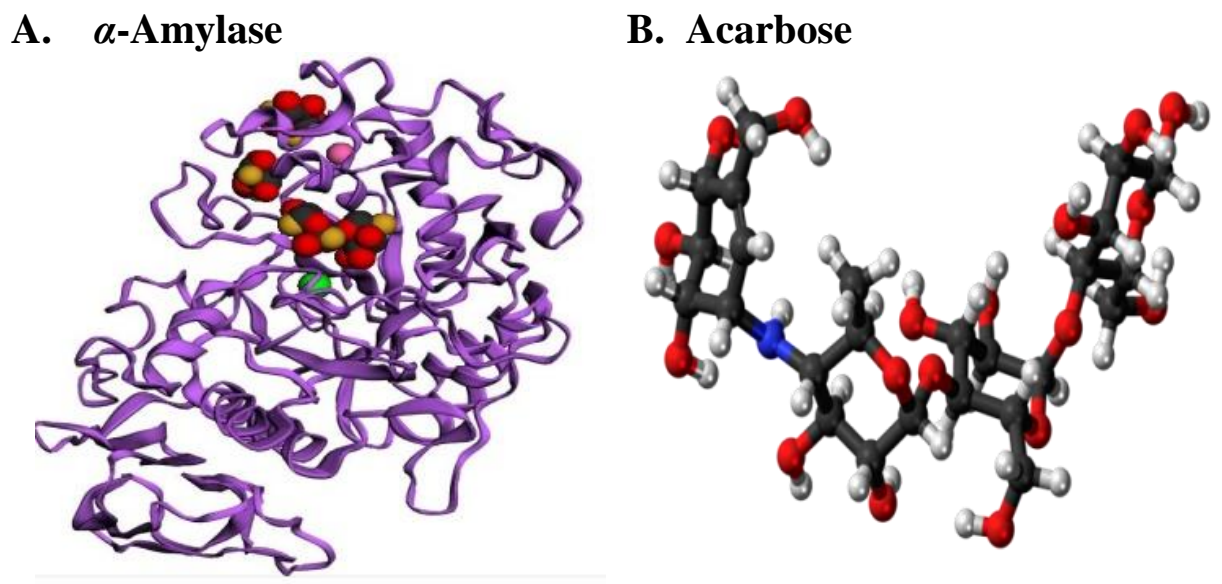

Figure 2. PDB Structures of target protein $(\mathbf{A})$ and standard drug (B).

\section{Results and Discussion}

\subsection{Chemistry}

For a long time different methods ${ }^{4,8,37-39}$ have been developed for the synthesis of wide variety of analogues, but Aldol condensation and Baker-venkataraman rearrangement are the most common methods to synthesize $(E)$-2-styrylchromoes from $o$-hydroxyacetopenones and cinnamaldehydes or cinnamic acids. The detailed synthetic methods, reactivity and biological activites of 2-styrylchromones were discussed by Silva etal ${ }^{40}$. In all above methods, majority of synthetic routs ${ }^{41-43}$ were multistep and also frequently involving protection-deprotection steps ${ }^{44}$. So the use of protecting groups makes the entire synthetic plan more complex since it requires at least two additional steps. At the same time, it is desirable to design synthetic procedure with high selectivity, high-yielding, clean and manipulatively easy.

Based on the above facts and literature survey, we focused on the use of PEG- $400^{45,46}$ which is an excellent solvent and, non-toxic, thermally stable, eco-friendly, recoverable and reusable ${ }^{47,48}$ reaction medium/condensing agent ${ }^{49-51}$.With the previous experience in the synthesis, we have started preparation of 2-styrylchromones using PEG-400 as a solvent cum condensing agent at $40-50{ }^{\circ} \mathrm{C}$ temperature followed by oxidative cyclization with iodine at $\approx 140{ }^{\circ} \mathrm{C}$.

In majority of cases iodine was used for oxidative cyclization in DMSO under different conditions to get flavones ${ }^{52}$ and other chromone derivatives ${ }^{40,53}$. Iodine was also used by Pinto ${ }^{54}$ and Silva for iodination of chromones to enhance their biological activities and also as condensing (dehydrating) agent to convert $\beta$-diketones into respective chromone derivatives. 
Before the use of PEG-400, we tried with Polyphosphoric acid (PPA) alone and also along with some solvents like ethanol, methanol etc. But the yield of products was very low even on long stirring for about several hours. Later we have carried out the optimization of reaction conditions (Table 1) with the starting materials and found that the products were obtained in good yield at high purity when the reaction was run under warm conditions in PEG-400 by using piperidine in trace amounts. Initially, we have prepared 1,5-diphenylpenta-2,4-dienones from $o$-hydroxy- acetophenones and connamaldehydes in the first step. These compounds were then converted into corresponding 2-styrylchromones by means of oxidative cyclization in PEG-400 using iodine as oxidizing agent, Scheme 1.

After following the above method for 2-3 compounds, just we have run the entire synthetic process in the same reaction vessel without isolation and purification of dienones. The completion of reaction for each corresponding product was scrutinized by TLC. Even the two steps were made to complete in the same vessel, there was no that much decrease in the yield of final products. The physical data of synthesized products was given in the Table 2 .

Initially, the synthesized compounds were identified by LCMS and IR spectrum. Of course 2D$\mathrm{NMR}^{55}$ is also useful in structural eiucidation, 1D-NMR spectroscopy which is the most powerful technique to illustrate the structure of organic compounds was used for the structural elucidation of 2styrylchromones. The pair of doublets at $\delta 6.81-7.03$ and 7.27-7.57 ppm represents two hydrogens of a double bond $\left(\mathrm{C}_{\alpha}-\mathrm{C}_{\beta}\right)$ of styryl group. The trans configuration of $\alpha$ and $\beta$ (vicinal) protons was established from the large coupling constants $\left(J_{\mathrm{H} \alpha}-J_{\mathrm{H} \beta},\right)$ at around $16 \mathrm{~Hz}$. The presence of $-\mathrm{OH}$ and $-\mathrm{OCH}_{3}$ at two benzene nuclei was distinguished by resonance peaks at $\delta 9.07-9.81 \mathrm{ppm}$ and 3.83-3.92 ppm respectively. The C-3 proton was highly shielded which gave a strong peak in the range of 6.29-6.34 $\mathrm{ppm}$. All these structural features were well supported by ${ }^{13} \mathrm{C}-\mathrm{NMR}$ spectra and the hydroxyl and methoxyl substituted aromatic carbons were easily identified by larger $\delta$ values about 149-163 ppm.

All the synthesized compounds were given an intensified molecular ion peak in negative ion mode at their respective molecular mass. The presences of various functional groups like $\mathrm{C}=\mathrm{O},-\mathrm{C}-\mathrm{O}-$ $\mathrm{C}-$ - $-\mathrm{OH},-\mathrm{OCH}_{3}$ were well recognized from their IR spectra.

\subsection{In Silico Molecular Docking Studies}

Based on important activities of some other chromones including 2-styrylchromones ${ }^{41,43,44,56,57-}$ ${ }^{62}$, the docking studies were performed for the synthesized compounds (20-31) to understand their binding interactions with the active site of the pancreatic $\alpha$-amylase enzyme (PDB ID: 3IJ8) using 1click docking online server tool. All most all the 12 compounds in this series were found to be active. From the docking results, it was observed that the compounds 24, 25, 26 and 28 were exhibited good binding energies $(-8.7,-8.8,-8.6$ and $-8.4 \mathrm{kcal} / \mathrm{mol})$ with the target enzyme, $\alpha$-amylase when compared with the reference drug $(-8.3 \mathrm{kcal} / \mathrm{mol})$ and were fitted in the active site of the target gene properly. Binding energies and bonding pose of ligands with target enzyme were presented in detail in Table 3. 2D ligand interactions of most active compounds $(\mathbf{2 8}, \mathbf{2 6}, \mathbf{2 4}$ and $\mathbf{2 5})$ with the target enzyme were shown in Table 4.

The compound $\mathbf{2 5}$ is the most active compound in this series and the binding mode shows that the chrome nucleolus and phenyl group of compound $\mathbf{2 5}$ forms $\pi$ - $\pi$ stacking with Trp59 and Tyr62 respectively. Additionally, the compound was also found to have hydrophobic interactions with Leu165, Leu162, Ala198, Tyr62, Trp59, Trp58 and Trp59. In compound 24, the chromone ring forms $\pi-\pi$ stacking with Trp59. Additionally, Leu165, Leu162, Ala198, Tyr62, Trp59 and Trp58 formed hydrophobic contacts with compound 24. The binding mode orientation of the compound 26 showed that the chromone ring forms $\pi-\pi$ stacking with Trp59. 
Table 1. Optimization of reaction conditions:

\begin{tabular}{cccccc}
\hline S.No. & Solvent & Reagent & Temp. $\left({ }^{\circ} \mathbf{C}\right)$ & Time $($ hrs.) & Yield (\%) \\
\hline 1 & PPA & - & RT & $3-4$ & ND \\
2 & EtOH & PPA & RT & 3 & Very poor \\
3 & MeOH & PPA & $40-50$ & $2-3$ & $10-20$ \\
4 & PEG-400 & - & RT & $3-5$ & ND \\
5 & PEG-400 & - & $90-100$ & 3 & $10-20$ \\
6 & PEG-400 & $\mathrm{Et}_{3} \mathrm{~N}$ & $40-50$ & $1-2$ & $25-30$ \\
7 & PEG-400 & Et $_{2} \mathrm{NH}$ & $40-50$ & $1-2$ & $\approx 30$ \\
8 & PEG-400 & Piperidine & $40-50$ & 0.5 & $85-87$ \\
\hline
\end{tabular}

Additionally, Ala198, Leu162, Leu165, Tyr62, Trp59 and Trp58 forms hydrophobic contacts with compound 26. In compound 28, the chromone ring forms $\pi$ - $\pi$ stacking with His 201 . Additionally, Val234, Ile235, Ala198, Tyr151, Leu162, Leu165, Tyr62, Trp59 and Trp58 form hydrophobic contacts with compound 24. Additionally, the oxygen atom of chrome ring forms hydrogen bond with Tyr 151 .

Table 2. Physical data of synthesized compounds

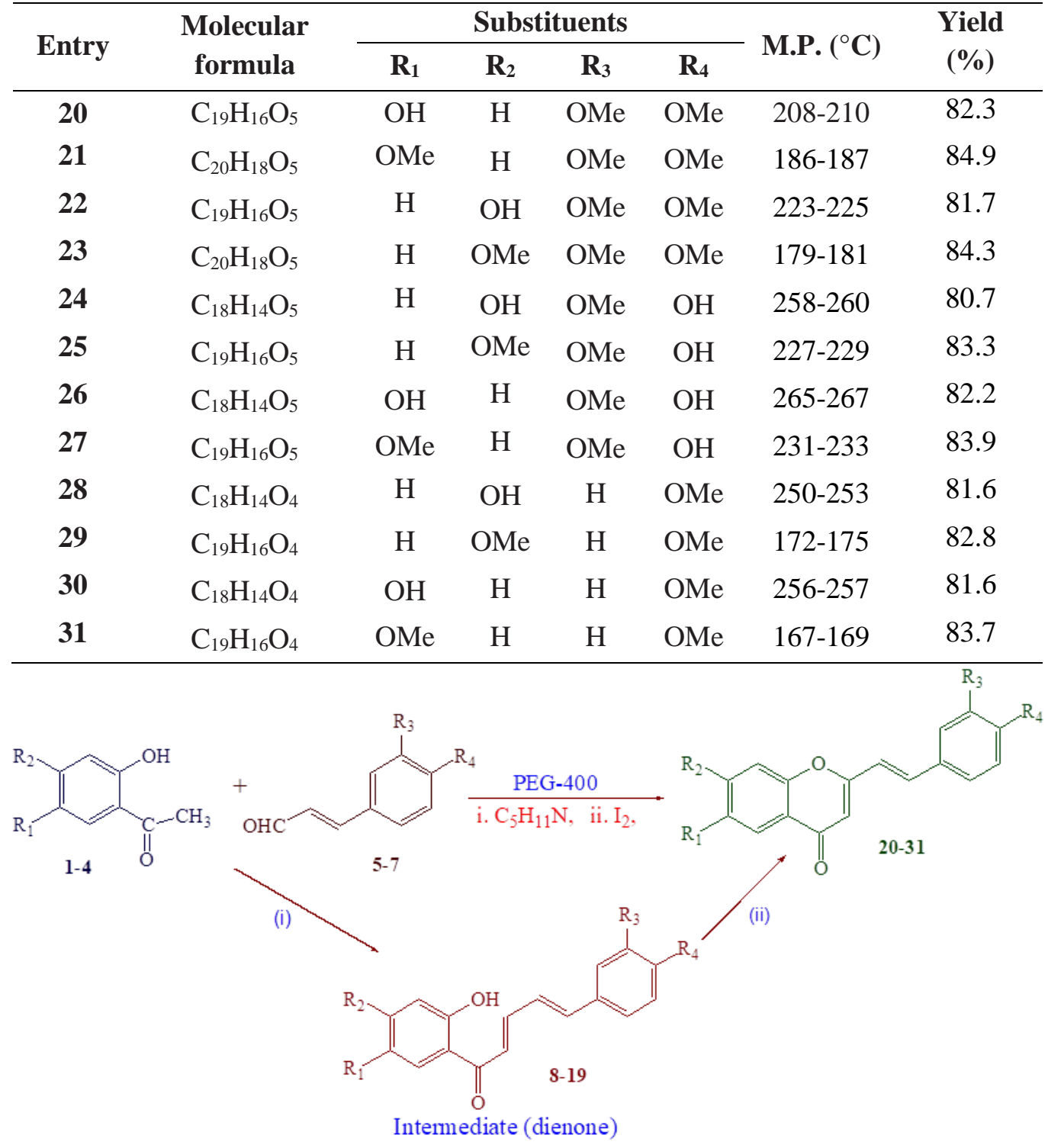

Scheme 1. Preparation of 2-styrylchromones

(i) PEG-400, $\mathrm{C}_{5} \mathrm{H}_{11} \mathrm{~N}, 40-50{ }^{\circ} \mathrm{C}, 0.5$ hrs. (ii) PEG-400, $\mathrm{I}_{2}, \approx 140{ }^{\circ} \mathrm{C}, 3-4 \mathrm{hrs}$ 
Table 3. Bonding interactions of the title lead compounds (20-31) and standard with $\alpha$-amylase

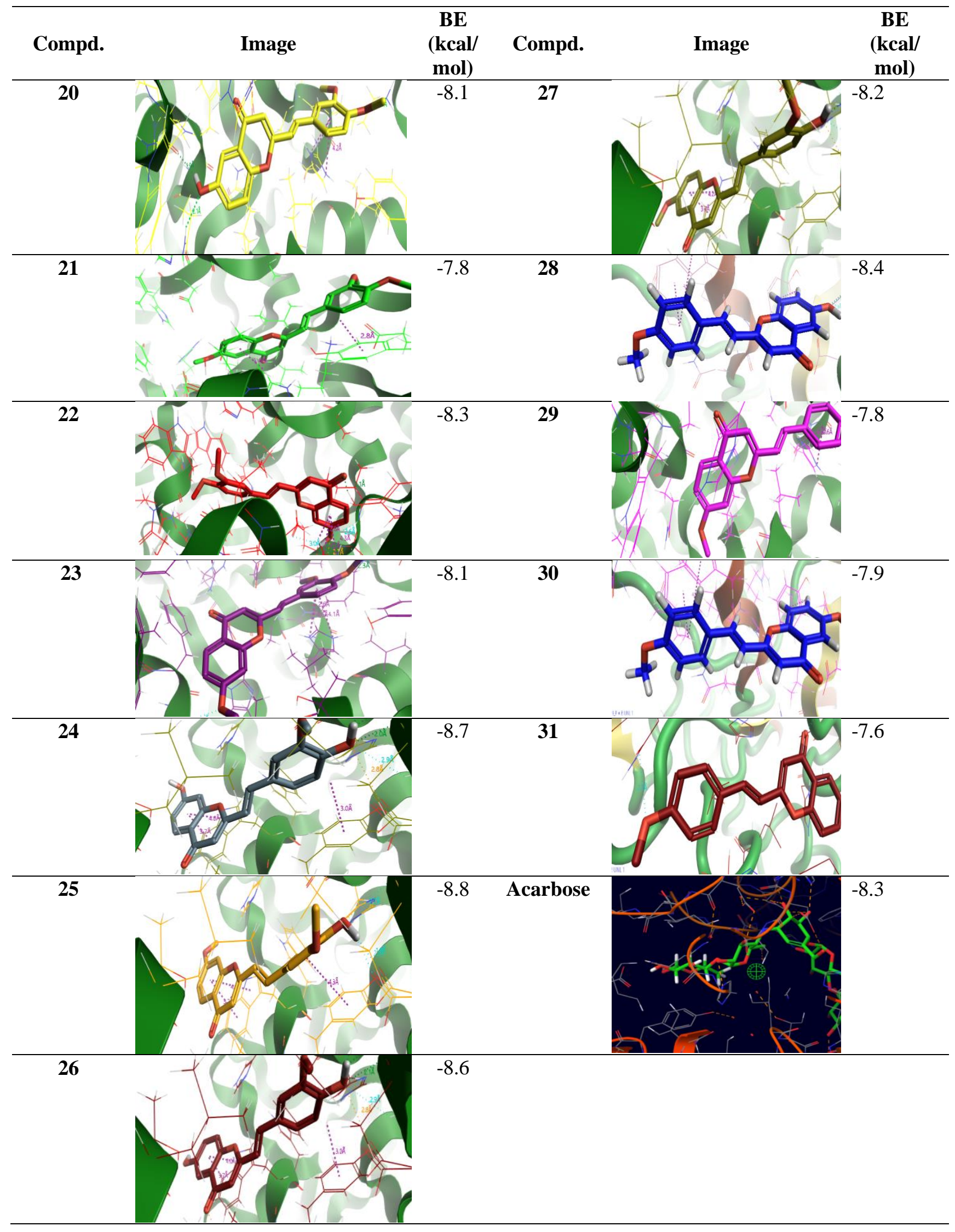


Table 4. 2D Lig Plot images of compounds $24,25,26$ and 28

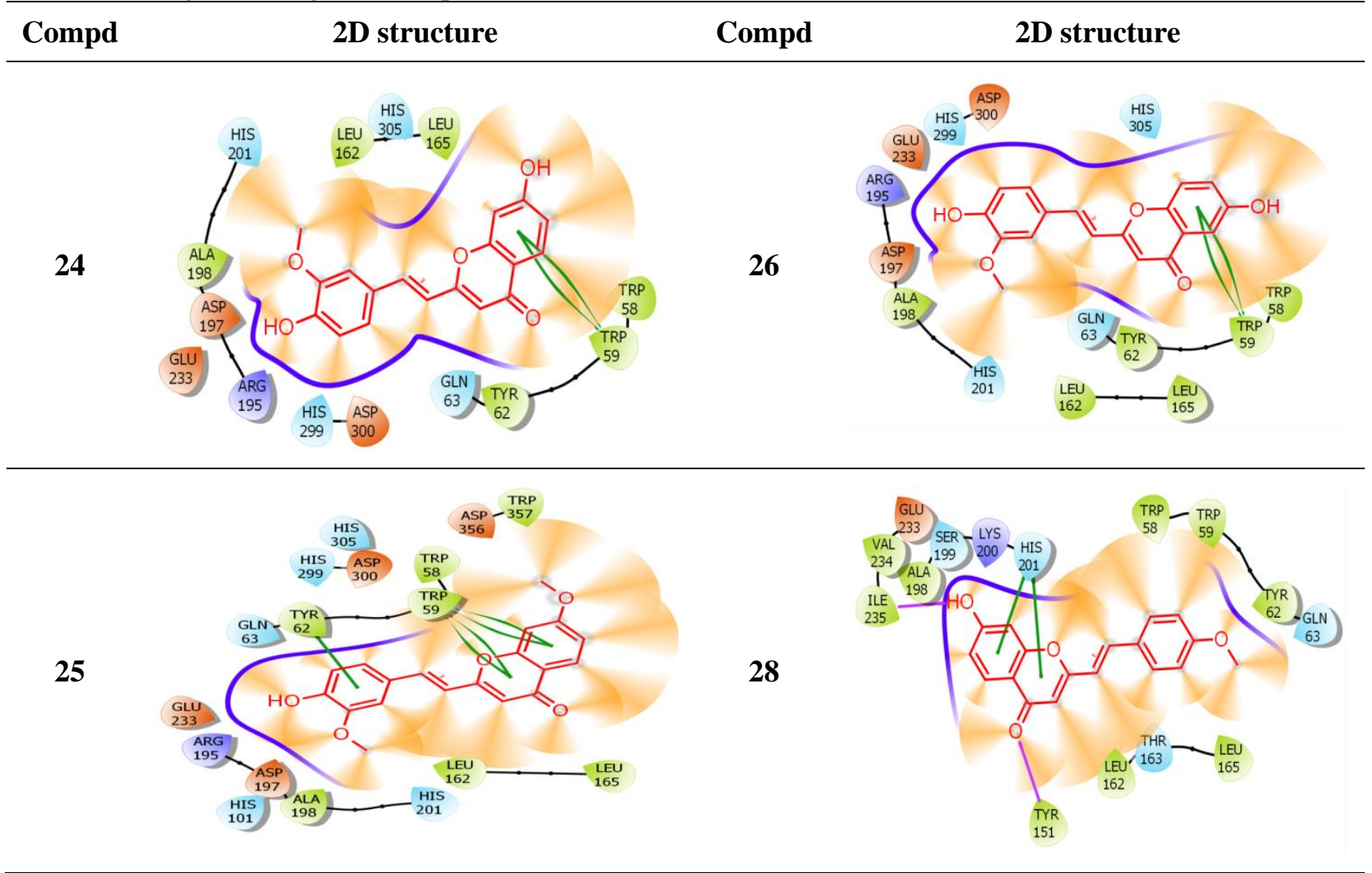

\section{Conclusion}

We have attempted a new way for the synthesis of 2-styrylchromones with PEG-400 in the presence of catalytic amounts of piperidine and iodine. The way, we proceeded was satisfactory in the completion of the reaction, yield and purity of final products. In silico molecular docking study was also performed for all the ligands against human $\alpha$-amylase enzyme. The study established that the compounds 24 (4',7-OH, 3'-OMe ), 25 (33',7-OMe, 4'-OH), 26 (4',6-OH, 3'-OMe) and 28 (4'-OMe, 7-OH) were exhibited higher binding energies $(-8.7,-8.8,-8.6$ and $-8.4 \mathrm{Kcal} / \mathrm{mol})$ with the target enzyme than the reference drug, acarbose $(-8.2 \mathrm{kcal} / \mathrm{mol})$ which shows that the synthesized compounds will become the promising next-generation anti-diabetic agents.

\section{Acknowledgements}

The authors are very thankful to department of Chemistry, Bapatla Engineering College, Bapatla on providing lab facility including some chemicals, and also thankful to Prof. W. Rajendra, Division of Molecular Biology, S.V. University, Tirupati, for their endless support in molecular docking studies and other activity studies. We extend our gratitude to N. Sudheer Kumar, Deptartment of English, BEC, for making necessary grammatical corrections.

\section{Supporting Information}

Supporting information accompanies this paper on http://www.acgpubs.org/journal/organiccommunications

\section{ORCID}

Priyadarsini Pullagura: 0000-0001-8309-1638

Madhava Rao Vallabhaneni: 0000-0002-0031-5200

Hanumatha Rao Addanki: 0000-0001-5371-5825 
Subramanyam Chennamsetty: 0000-0002-0422-6096

Ranganayakulu Yenisetty: 0000-0003-1237-3819

\section{References}

[1] Sugita, Y.; Takao, K.; Uesava, Y.; Nagai, J.; Lijima, Y.; Sano, M.; and Sakagami, H. Development of newly synthesized chromone derivatives with high tumor specificity against human oral squamous cell carcinoma. Medicines 2020, 7(9), 50, (18 pages).

[2] Takao, K.; Endo, S.; Nagai, J.; Kamauchi, H.; Takemura, Y.; Uesawa, Y.; Sugita, Y. 2-Styrylchromone derivatives as potent and selective monoamine oxidase B inhibitors. Bio-org. Chem. 2019, 92, 1-10.

[3] Uesawa, Y.; Nagai, J.; Shi, H.; Sakagami, H.; Bandow, K.; Tomomura, A.; Tomomura, M.; Endo, S.; Takao, K.; Sugita, Y. Quantitative structure-cytotoxicity relationship of 2-styrylchromones. Anticancer Res. 2019, 39, 6489-6498.

[4] Gomes, A.; Freitas, M.; Fernandes, E.; Lima, J.L.F.C. Biological activities of 2-styrylchromones. Mini Rev Med. Chem. 2010, 10, 1-7.

[5] Keri, R.S.; Budagumpi, S.; Pai, R.K.; Balakrishna, R.G. Chromones as a privileged scaffold in drug discovery: a review. Eur. J. Med.Chem. 2014, 78, 340-374.

[6] Gaspar, A.; Matos, M.J.; Garrido, J.; Uriarte, E.; Borges, F. Chromone: a valid scaffold in medicinal chemistry. Chem. Rev. 2014, 114, 4960-4992.

[7] Sharma, K.S.; Kumar, S.; Chand, K.; Kathuria, A.; Gupta, A.; Jain, R. An update on natural occurrence and biological activity of chromones. Curr. Med. Chem. 2011, 18, 3825-3852.

[8] Silva, A.M.S.; Pinto, C.G.A.; Cavaleiro, J.A.S.; Levai,A.; Patonay, A. Synthesis and reactivity of styrylchromones. Arkivoc 2004, vii, 106-123.

[9] Santos, C.M.M.; Silva, A.M.S. An Overview of 2-Styrylchromones: natural occurrence, synthesis, reactivity and biological properties. Eur. J. Org. Chem. 2017, 22, 3115-3133.

[10] Singhi, M. An efficient synthesis of 3-hydroxy-2-styryl chromones: potent anti-rhinovirus and antiNorovirus Agents, J. Pharm. Res. 2011, 4(9), 3040-3041.

[11] Ujwala,B.; Priyaarsini, P.; Madhava Rao, V. Synthesis and bioactivity evaluation of 2-styrylchromones. Int. J. Pharma Bio Sci. 2013, 4(1), 199-206.

[12] Pinto, J.; Silva, V.L.M.; Silvla, A.M.G.; Silva, A.M.S. Synthesis of (e)-2-styrylchromones and flavones by base-catalyzed cyclodehydration of the appropriate $\beta$-diketones using water as solvent. Molecules 2015, 20, 11418-11431.

[13] Sharma, D.; Makrandi, J.K. A green synthesis of 2-phenyl/2-styrylchromones under solvent-free conditions using grinding technique. Green Chem. Lett. \& Rev. 2009, 2(3), 157-159.

[14] Tiwari, S. V.; Seijas, J. A.; Vazquez-Tato, M. P. Ionic liquid-promoted synthesis of novel chromonepyrimidine coupled derivatives, antimicrobial analysis, enzyme assay, docking study and toxicity study. Molecules 2018, 23(2), 1-23.

[15] Tome, S.M.; Silva, A.M.S. and Santos, C.M.M. Synthesis and transfermation of halochromones. Current Org. Synth. 2014, 11(3), 317-341.

[16] Tawfik, H.A.; Ewies, E.F.; EL-Hamouly, W.S. Synthesis of chromones and their applications during last ten years. Int. J. Res. Pharm. Chem. 2014, 4(4), 1046-1085.

[17] Talhi, O.; Brodziak-Jarosz, L.; Panning, J.; Orlikova, B.; Zwergel, C. ; Tzanova, T.; Philippot, S.; Pinto, D. C. G. A. ; Paz, F. A. A. ; Gerhauser, C. ; Dick, T. P. ; Jacob, C.; Diederich, M.; Bagrel, D. ; Kirsch, G. ; Silva, A. M. S. One-pot synthesis of benzopyran - 4 - ones with cancer preventive and therapeutic potential. Eur. J. Org. Chem. 2016, 2016(5), 965-975.

[18] Santos, C. M. M. ; Silva, A. M. S.; Cavaleiro, J. A. S. Synthesis of new hydroxy-2-styrylchromones. Eur. J. Org. Chem. 2003, 2003(23), 4575-4585.

[19] Baptista, F. R.; Pinto, D. C. G. A.; Silva, A. M. S. Towards the synthesis of platachromone b, a bioactive natural prenylated (e)-2-styrychromone. Synlett 2014, 25(08), 1116-1120.

[20] Gomes, A; Fernandes, E.; Silva, A.M.S.; Santos, C.M.M.; Pinto, D.C.G.A.; Cavaleiro, J.A.S. Lima J.L.F.C., 2-Styrylchromones: novel strong scavengers of reactive oxygen and nitrogen species. Bio-org. Med. Chem. 2007, 15(18), 6027-6036.

[21] Marinho, J.; Pedro, M.; Pinto, D.C.G.A.; Silva, A.M.S.; Cavaleiro, J.A.S.; Sunkel, C.E.; Nascimento, M.S.J. 4'-Methoxy-2-styrylchromone a novel microtubule-stabilizing antimitotic agent. Biochem. Pharmacol. 2008, 75(4), 826-835.

[22] Shawa, A.Y.; Chang, C.Y.; Liau, H.H.; Lu, P.J.; Chen, H.L.; Yang, C.N.; Li, H.Y. Synthesis of 2styrylchromones as a novel class of antiproliferative agents targeting carcinoma cells. Eur. J. Med. Chem. 2009, 44(6), 2552-2562.

[23] Brion, D.; Le Baut, G.; Zammatio, F.; Pierre, A.; Attasi, G.; Belachm, L. Preparation of 2-styryl-4chromanones as anticancer agents. Chem. Abstr. 1991, 116, 106092k. 
[24] Desideri, N.; Conti, C.; Mastromarino, P.; Mastropaolo, F. Synthesis and anti-rhinovirus activity of 2styrylchromones. Antivir. Chem. Chemotherapy 2000, 11(6), 373-381.

[25] Bozdag-Dundar, O; Verspohl, E.J.; Waheed, A.; Ertan, R. Synthesis and anti-diabetic activity of some new furochromonyl-2,4-thiazolidinediones. Arzneimittelforschung 2003, 53, 831-836.

[26] Bozdag-Dundar, O.; Ceylan-Ünlüsoy, M.; Verspohl, E.J.; Ertan, R. Synthesis and antidiabetic activity of some new chromonyl-2,4-thiazolidinediones. Arzneimittelforschung 2007, 57, 532-536.

[27] Gerwick, W.H.; Lopez, A.; Van Duyne, G.D.; Clardy, J.; Ortiz, W.; Baez, A. Hormothamnione, a novel cytotoxic styrylchromone from the marine cyanophyte-harmothaminon enteromorphides grunow. Tetrahed. Lett. 1986, 27, 1979-1982.

[28] Gerwick, W.H. 6-Desmethoxyhormothamnione, a new cytotoxic styrylchromone from the marine cryptophyte Chrysophaeum taylori. J. Nat. Prod. 1989, 52(2), 252-256.

[29] Yoon, J. S.; Lee, M. K.; Sung, S. H.; Kim, Y. C. Neuroprotective 2-(2-phenylethyl)chromones of Imperata cylindrica. J. Nat. Prod. 2006, 69(2), 290-291.

[30] Yang, L.; Qiao, L. Xie,; D.; Yuan, Y.; Chen, N.; Dai, J.; Guo, S. 2(2-phenylethyl)chromones from Chinese eaglewood. Phytochemistry 2012, 76, 92-97.

[31] Yang, C.H.; Yang, Y.; Liu, J.H. Platachromone A-D: Cytotoxic 2-styrylchromones from the bark of Platanus $\times$ acerifolia (Aiton) Willd. Phytochem. Lett. 2013, 6, 387-391.

[32] Jung, H. J.; Jung, H. A.; Min, B.-S; Choi, .; J. S. Anticholinesterase and $\beta$-site amyloid precursor protein cleaving enzyme 1 inhibitory compounds from the heartwood of Juniperus chinensis. Chem. Pharm. Bull. 2015, 63(11), 955-960.

[33] Chaniad, P.; Wattanapiromsakul, C.; Pianwanit, S.; Tewtrakul, S., Anti-HIV-1 integrase compounds from Dioscorea bulbifera and molecular docking study, Pharm. Biol.2016, 54(6), 1077-1085.

[34] Trott, O.; Olson, A.J. AutoDock Vina: improving the speed and accuracy of docking with a new scoring function, efficient optimization, and multithreading. J. Comput. Chem. 2010, 31(2), 455-461.

[35] Altaff, SK. Md.; Raja Rajeswari, T.; Subramanyam, Ch. Synthesis, $\alpha$-amylase inhibitory activity evaluation and In silico molecular docking study of some new phosphoramidates containing heterocyclic ring. Phosphorus, Sulfur Silicon Relat. Elem. 2020, 1-9. DOI: https://doi.org/10.1080/10426507.2020.1845679.

[36] Madhu Kumar Reddy, K.; Peddanna, K.; Varalakshmi, M.; Bakthvatchala Reddy, N.; Sravya, G.; Grigory, V.Z.; Suresh Reddy, C. Ceric ammonium nitrate (CAN) catalyzed synthesis and $\alpha$-glucosidase activity of some novel tetrahydropyridine phosphonate derivatives. Phosphor. Sulfur Silicon Rel. Element. 2019, 194(8), 812-819.

[37] Price, W.A.; Silva, A.M.S.; Cavaleiro, J.A.S. 2-Styrlchromones: biological action, synthesis and reactivity. Heterocycles 1993, 36(11), 2601-2612.

[38] Pinto, D.C.G.A.; Silva, A.M.S.; Cavaleiro, J.A.S. A Convenient synthesis of new (e)-5-hydroxy-2styrylchromones by modifications of the baker-venkataraman method. New J. Chem. 2000, 24(2), 85-92.

[39] Gomes. A.; Neuwirth, O.; Freitas, M.; Couto, D.; Ribiero, D.; Figueiredo, A.G.P.R.; Silva, A.M.S.; Seixas, R.S.G.R.; Pinto, D.C.G.A.; Tome, A.C. Synthesis and antioxidant properties of new chromone derivatives. Bioorg. Med. Chem. 2009, 17, 7218-7226.

[40] Silva, A. M. S.; Pinto, D. C. G. A.; Cavaleiro, J. A. S.; Levai, A; Patonay, T. Synthesis and reactivity of styrylchromones. ARKIVOC 2004, vii 106-123.

[41] Madhava, Rao V.; Ujwala, B.; Priyadarshini, P.; Krishna, Murthy P. Synthesis, antioxidant and antimicrobial activity of three new 2-styrylchromones and their analogues. Der Pharm. Chem. 2016, 8(7), 1-6.

[42] Makrandi, J. K.; Vandna, K. A convenient synthesis of 2-styrylchromones by modified BakerVenkataraman transformation using phase transfer catalysis. Synthetic Commun. 1989, 19(11-12), 19191922.

[43] Safitri, B. V.; Kristanti, A. N.; Kautsar Ul Haq, H. S.; Hendriyanto, D. Synthesis of 2-styrylchromones: In vitro and in silico anticancer activity evaluation. J. Appl Pharmaceut. Sci. 2021, 11 (01), 121-128.

[44] Vasu, K.; Harikrishna, N.; Nagaraju, K.; Venkata Rao, C. Synthesis of 2-styrylchromones and cytotoxicity evaluation. Pharm. Sinica 2014, 5(4), 40-46.

[45] Chnadrasekhar, S.; Narasihmulu, Ch.; Sultana, S.S.; Reddy, N.R. Osmium tetroxide in poly(ethylene glycol) (PEG): a recyclable reaction medium for rapid asymmetric dihydroxylation under Sharpless conditions. Chem. Commun., 2003, 2003(14), 1716-1717.

[46] Choudary, B.M.; Jyothi, K.; Madhi, S.; Kantam, M.L. Allylation of aldehydes, aldimines and ring opening of terminal aromatic epoxides by scandium triflate using polyethylene glycol (peg) as an efficient recyclable medium. Synlett 2004, 2004(2), 231-234.

[47] Jayapal, M.R.; Sreenivasa Prasad, K.and Sreedhar, N.Y. Synthesis and characterization of 2,6-dihydroxy substituted chalcones, using peg-400 as a recyclable solvent. J. Pharm. Sci. Res. 2010, 2(8), 450-458. 
[48] Gupta, A. K.; Bharadwaj, M. and Mehrotra, R. Eco-friendly polyethylene glycol-400 as a rapid and efficient recyclable reaction medium for the synthesis of anticancer isatin-linked chalcones and their 3hydroxy precursor. J. Heterocyclic Chem. 2019, 56, 703-709.

[49] Tanemura, K.; Suzuki, T.; Nishida, Y.; Horaguchiy, T. Aldol condensation in water using polyethylene glycol 400. Chem. Lett. 2005, 34(4), 576-577.

[50] Sudhakar, D.; Madhava Rao, V.; Siddhaiah, V.; Venkata Rao, C. Facile polyethylene glycol (PEG-400) promoted synthesis of oximes. Org. Chem. An Ind. J. 2010, 6(1), 63-65.

[51] Zhong, X.; Dou, G.; Wang, D. Polyethylene Glycol (PEG-400): An efficient and recyclable reaction medium for the synthesis of pyrazolo[3,4-b]pyridin-6(7h)-one derivatives. Molecules 2013, 18(11), 1313913147.

[52] Patel, S.; Shah, U. Synthesis of flavones from 2-hydroxy acetophenone and aromatic aldehyde derivatives by conventional methods and green chemistry approach. Asian J Pharm. Clin. Res. 2017, 10(2), 403-406.

[53] Santos, C. M. M.; Silva, V. L. M. and Silva, A. M. S. Synthesis of chromone-related pyrazole compounds. Molecules 2017, 22(10), 1665, 1-47.

[54] Pinto, D. C. G. A. and Silva, A. M. S. Molecular iodine in the synthesis of chromone-type compounds. Curr. Org. Synth. 2012, 9(4), 561-572.

[55] Momin, M.; Ramjugernath, D. and Koorbanally, N. A. Structure elucidation of a series of fluoro-2styrylchromones and methoxy-2-styrylchromones using 1D and 2D NMR spectroscopy. Magn. Reson. Chem. 2014, 52(9), 521-529.

[56] Madhava Rao, V.; Jewahar Babu, P. and Venkata Rao, C. Antifungal and antibacterial activity of synthesized 2-styrylchromones. Int. J. Pharm. Res. Develop. 2013, 5(01), 037 - 045.

[57] Takao, K.; Hoshi, K.; Sakagami, H.; Shi, H.; Bandow, K.; Nagai, J.; Uesawa, Y.; Tomomura, A.; Tomomura, M.; Sugita, Y. Further quantitative structure-cytotoxicity relationship analysis of 3styrylchromones. Anticancer Res. 2020, 40, 87-95.

[58] Takao, K.; Yahagi, H.; Uesawa, U.; Sugita, Y. 3-(E)-Styryl-2H-chromene derivatives as potent and selective monoamine oxidase B inhibitors. Bioorg. Chem. 2018, 77, 436-442.

[59] Makhaeva, G. F.; Boltneva, N. P.; Lushchekina, S. V.; Beloglazkin A.A.; Borisov, R.S.; Richardson, R.J. Synthesis, molecular docking, and biological activity of 2-vinyl chromones: Toward selective butyrylcholinesterase inhibitors for potential Alzheimer's disease therapeutics. Bioorg. Med. Chem. 2018, 26(16), $4716-4725$.

[60] Sousa, J. L. C.; Silva, A. M. S. Microwave-assisted tandem reactions towards a new synthesis of cyclohepta[b]chromene-9,11-diones. Synlett 2017, 28, 316-322.

[61] Koichi, T.; Ryo, I. and Yoshiaki S. Synthesis and biological evaluation of 3-styrylchromone derivatives as free radical scavengers and $\alpha$-glucosidase inhibitors. Chem.Pharm.Bull. 2014, 62(8), 810-815.

[62] Li, F.; Wu, J.-J., and Wang, J. Synthesis and pharmacological evaluation of novel chromone derivatives as balanced multifunctional agents against Alzheimer's disease. Bioorg. Med. Chem. 2017, 25(14), $3815-$ 3826.

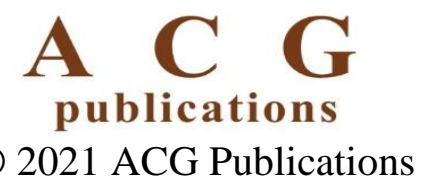

